University of Nebraska - Lincoln

DigitalCommons@University of Nebraska - Lincoln

Martin Centurion Publications

Research Papers in Physics and Astronomy

$11-2007$

\title{
Harmonic holography: a new holographic principle
}

Ye Pu

California Institute of Technology, yepu@sunoptics.caltech.edu

Martin Centurion

niversity of Nebraska - Lincoln, martin.centurion@unl.edu

Demetri Psaltis

École Polytechnique Fédérale de Lausanne

Follow this and additional works at: https://digitalcommons.unl.edu/physicscenturion

Part of the Physics Commons

$\mathrm{Pu}, \mathrm{Ye}$; Centurion, Martin; and Psaltis, Demetri, "Harmonic holography: a new holographic principle" (2007). Martin Centurion Publications. 3.

https://digitalcommons.unl.edu/physicscenturion/3

This Article is brought to you for free and open access by the Research Papers in Physics and Astronomy at DigitalCommons@University of Nebraska - Lincoln. It has been accepted for inclusion in Martin Centurion Publications by an authorized administrator of DigitalCommons@University of Nebraska - Lincoln. 


\title{
Harmonic holography: a new holographic principle
}

\author{
Ye Pu, ${ }^{1, *}$ Martin Centurion, ${ }^{1,2}$ and Demetri Psaltis ${ }^{1,3}$ \\ ${ }^{1}$ Department of Electrical Engineering, California Institute of Technology, Pasadena, California 91125, USA \\ 2Laboratory for Attosecond and High-Field Physics, Max-Planck-Institut für Quantenoptik, Hans-Kopfermann-Strasse 1, \\ D-85748 Garching, Germany \\ 3École Polytechnique Fédérale de Lausanne (EPFL), Laboratoire d'optique, STI IOA LO, BM 4102 (Bâtiment BM), \\ Station 17, CH-1015 Lausanne, Switzerland \\ *Corresponding author: yepu@sunoptics.caltech.edu
}

Received 7 May 2007; accepted 5 July 2007;

posted 26 September 2007 (Doc. ID 82771); published 29 November 2007

\begin{abstract}
The process of second harmonic generation (SHG) has a unique property of forming a sharp optical contrast between noncentrosymmetric crystalline materials and other types of material, which is a highly valuable asset for contrast microscopy. The coherent signal obtained through SHG also allows for the recording of holograms at high spatial and temporal resolution, enabling whole-field four-dimensional microscopy for highly dynamic microsystems and nanosystems. Here we describe a new holographic principle, harmonic holography $\left(\mathrm{H}^{2}\right)$, which records holograms between independently generated second harmonic signals and reference. We experimentally demonstrate this technique with digital holographic recording of second harmonic signals upconverted from an ensemble of second harmonic generating nanocrystal clusters under femtosecond laser excitation. Our results show that harmonic holography is uniquely suited for ultrafast four-dimensional contrast microscopy. (C) 2008 Optical Society of America OCIS codes: $\quad 090.0090,090.1000,180.6900,190.4180,190.7220$.
\end{abstract}

\section{Introduction}

One of the grand open challenges in modern science is to probe and understand the mechanism and dynamics of biological processes inside living cells at the molecular level. Besides the highly complex threedimensional (3D) structures spanning a large range of length scale [1], living organisms by their nature are highly dynamic: Molecular processes such as protein, DNA, and RNA conformation changes take place on a time scale ranging from $100 \mathrm{fs}$ to $100 \mathrm{~s}[2,3]$ while the organisms move and metabolize. Essentially, probing these processes requires taking $3 \mathrm{D}$ microscopic pictures in a rapid succession with high sensitivity and specificity, i.e., a four-dimensional (4D) microscope is needed.

Since holography was invented 60 years ago [4], an aberration-free, 3D microscope has long been hoped for. In fact, soon after Leith and Upatniek demonstrated the first 3D image by holography [5], holographic microscopy was proposed [6]. With the recent

0003-6935/08/040A103-8\$15.00/0

(C) 2008 Optical Society of America advent of digital holography [7,8], holographic 3D microscopy received renewed and rapidly increasing attention [9-16]. Holography is a "whole-field" technique, which means recording all $3 \mathrm{D}$ pixels simultaneously in one laser shot without scanning, an extremely valuable asset for biomedical imaging. When combined with modern high-repetition rate pulsed laser and fast imaging devices, a time sequence of consecutive $3 \mathrm{D}$ images can be captured, forming a 4D microscope. Indeed, successful 4D holographic imaging has been achieved recently in the context of fluid velocity measurement [17].

Despite the technical advancements, holographic microscopes are not widely deployed in biomedical research because of the lack of specificity. A holographic microscope would capture all scattering entities in the viewing field faithfully but indiscriminately. On the other hand, the signals of interest (often from small nanostructures like protein molecules) are usually very weak and buried in the strong ambient scatterings from much larger organelles.

A key to achieving the specificity required for molecular biomedical imaging is creating a contrast be- 
tween the useful signal and the ambient scatterings. Contrast imaging is routinely achieved through tagging the molecule or nanostructure of interest with fluorescent agents, such as fluorescent dyes, green fluorescent proteins, and quantum dots. By converting the light signal into a different frequency, the unwanted ambient scattering can be easily removed with proper optical filters. However, the incoherent fluorescence signal lacks the capability of $3 \mathrm{D}$ representation, and the finite scanning speed constrains these techniques to mostly two dimensions. Attempts to extend fluorescence microscopy into three spatial dimensions over time (4D microscopy) [18] result in a great sacrifice of framing time and are thus incapable of capturing dynamic events.

Here we show that the new holographic principle described below, namely, harmonic holography $\left(\mathrm{H}^{2}\right)$, provides an ideal solution to meet the ultimate need for dynamic 4D imaging with high contrast and ample spatial and temporal resolution.

\section{Harmonic Holography}

In our new holographic scheme, we use the second harmonic generation (SHG) process to achieve contrast imaging. When a nanocrystal of a noncentrosymmetric structure (a crystal structure that lacks an inversion center) is under the excitation of an intense optical field $\mathbf{E}(\omega)$, the polarization $\mathbf{P}(\omega)$ of the nanocrystal is described by a Taylor expansion series:

$$
\begin{aligned}
\mathbf{P}(\omega)= & \chi^{(1)} \cdot \mathbf{E}(\omega)+\chi^{(2)} \cdot \mathbf{E}(\omega) \cdot \mathbf{E}(\omega) \\
& +\chi^{(3)} \cdot \mathbf{E}(\omega) \cdot \mathbf{E}(\omega) \cdot \mathbf{E}(\omega)+\cdots,
\end{aligned}
$$

where the coefficient $\chi^{(n)}$ is the $n$ th-order susceptibilities of the crystal material. The second-order response, $\chi^{(2)} \cdot \mathbf{E} \cdot \mathbf{E}$, gives rise to a radiation at exactly twice the frequency of the pumping field. The capability of generating second harmonic radiation is specific to materials with noncentrosymmetric crystalline structures only, and $\chi^{(2)}$ vanishes for all other types of material. Therefore a sharp contrast is formed when particles of noncentrosymmetric structures are dispersed in a medium of other species, pumped at a fundamental frequency, and imaged at the second harmonic frequency. In contrast to fluorescence, a second harmonic signal is coherent, enabling holography with contrast at doubled spatial resolution. Additionally, SHG is an ultrafast process, permitting the probe of dynamics at a femtosecond level. With only a few exceptions [19], biological cells and tissues are incapable of generating endogenous second harmonic emissions. Therefore the new scheme described here provides a sound basis for a new type of contrast microscopy with enormous potential in molecular biomedical imaging.

Figure 1 illustrates the principle of harmonic holography $\left(\mathrm{H}^{2}\right)$. In this scheme, an intense laser pulse at frequency $\omega$ is delivered to a group of second harmonic generating nanocrystals, which are tagged to specific parts of the system under investigation. The nanocrystals emit scattering signals at both the fundamental

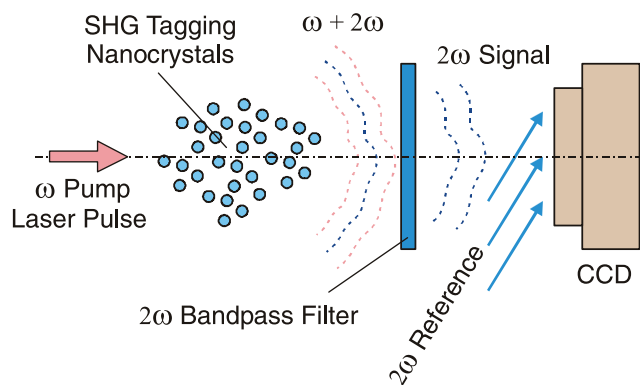

Fig. 1. (Color online) Principle of harmonic holography. An intense pump laser pulse of frequency $\omega$ is sent to a group of SHG tagging nanocrystals that scatter both $\omega$ and $2 \omega$ signals. The bandpass filter rejects the scatterings and the pump pulse at $\omega$ while passing signals at $2 \omega$. An independently frequency-doubled reference interferes with the $2 \omega$ signals at the CCD camera and forms a hologram. Structures that are not capable of generating second harmonic signals only scatter at $\omega$ and will not be recorded on the hologram.

$(\omega)$ and the second harmonic ( $2 \omega)$ frequencies. A bandpass filter centered at $2 \omega$ rejects the pumping and scattered light at frequency $\omega$ but transmits the $2 \omega$ signal to the charge-coupled device (CCD) sensor. A reference beam at frequency $2 \omega$, independently generated from the same pump laser pulse, is also delivered to the CCD sensor, and a hologram is thus formed. The coherence of the SHG process guarantees that the scattered $2 \omega$ signals have a deterministic, static phase relationship with the $2 \omega$ reference. It is worth noting that our scheme is fundamentally different from holography obtained in nonlinear crystals [20], where contrast is not achieved.

Fast, 3D imaging presents additional challenges. The second harmonic emissions from nanocrystals are usually extremely weak, often only a few photons per pulse. Furthermore, high numerical aperture (NA) lenses and objectives are often only optimized for one imaging plane, resulting in substantial aberrations when working with a volume of finite depth. In addition, fast image sensors with small pixels required in $\mathrm{H}^{2}$ are typically much noisier than slower ones with larger pixels. Therefore an implementation of $\mathrm{H}^{2}$ will seemingly encounter a serious signal to noise ratio (SNR) problem. However, the use of holography is able to address these issues simultaneously. Experiments $[12,21]$ have shown that objective aberrations can be numerically canceled or compensated in holography to obtain diffraction-limited imaging performance. Moreover, the reference used in holography serves as a coherent bias that is much higher than the device noise in the imaging sensor, leading to a shot noise-limited performance in the low photon count regime [22].

\section{Experimental Setup}

Figure 2 shows the experimental setup for $\mathrm{H}^{2}$. Femtosecond laser pulses of $2 \mathrm{~mJ}$ energy, $150 \mathrm{fs}$ full width at half-maximum (FWHM) duration, and $810 \mathrm{~nm}$ wavelength (repetition rate $10 \mathrm{~Hz}$ ) are split into a pump and a reference beam at beam splitter BS1. 


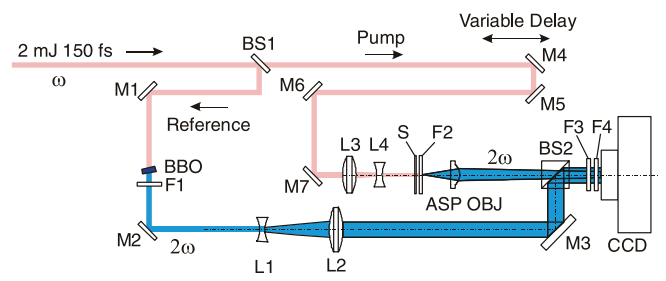

Fig. 2. (Color online) Experimental setup for harmonic holography. Femtosecond laser pulses of $2 \mathrm{~mJ}$ energy and $150 \mathrm{fs}$ pulse width FWHM is split into a pump and a reference beam at BS1. After a variable delay line, the pump carrying the major portion of the energy is shrunk by 3:1 with a reverse telescope (L3, L4) for higher intensity and sent to the sample containing SHG nanocrystal clusters. The second harmonic signals scattered from the nanocrystal clusters are collected by the aspheric objective and steered to the CCD camera. The reference, frequency doubled through a BBO crystal, interferes with the signal at the CCD plane and forms a hologram. BS1, BS2, beam splitters; L1, L2, lenses; ASP OBJ, aspheric objective; M1-M8, mirrors; F1-F4, filters; S, sample.

Approximately $1 \mathrm{~mJ}$ of the energy is delivered to the sample containing randomly dispersed SHG imaging targets. After the boost in intensity through the reversed 3:1 telescope L3 and L4, the pump intensity at the sample site is approximately $1 \times 10^{11} \mathrm{~W} / \mathrm{cm}^{2}$. While the intense pump beam at the fundamental frequency is rejected by the filter F2, the weak second harmonic radiations form the samples (the object wave) are picked up by the aspheric objective (N.A. $=0.5)$ at a magnification of $25 \times$. The reference, independently frequency doubled through a $\beta$-barium borate (BBO) crystal, meets and interferes with the signal at the beam splitter BS2 to form holograms that are captured by the CCD camera. The optical path lengths of the two second harmonic waves are carefully matched by the variable delay line.

We use clusters of $100 \mathrm{~nm} \mathrm{BaTiO}_{3}$ nanocrystals as the imaging target. Although tetragonal phase $\mathrm{BaTiO}_{3}$ has an excellent nonlinear coefficient $\chi^{(2)}[23]$, the unprocessed nanocrystals are in a centrosymmetric cubic crystal structure and are unable to produce second harmonic responses. To obtain tetragonal phase crystals, we sinter the nanocrystals at $1000{ }^{\circ} \mathrm{C}$ for $1 \mathrm{~h}$ and rapidly cool them in deionized water. While the sintering converts the crystal structures into tetragonal, large clusters result from such a process with a broad variation in size, as revealed by the scanning electronic microscopy (SEM) images shown in Fig. 3. For better control of the number density of particles, we collect the larger clusters through sedimentation and disperse them randomly on a microscope coverslip. Because of the "random phasematching" condition, we expect to see specklelike, randomly located spots within the extent of each cluster at the second harmonic frequency.

The position of the sample is adjustable. We capture a "direct" (on-focus) image when the reference is shut off and the sample is positioned at the imaging focal plane. A hologram, on the other hand, is obtained when the sample is moved off focus by a short

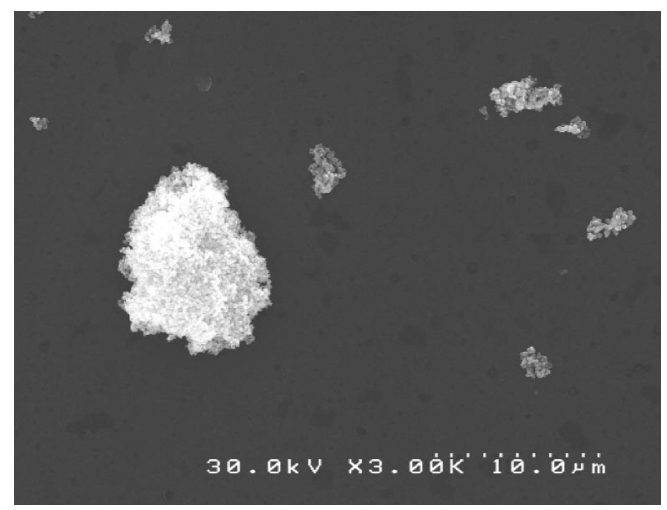

Fig. 3. SEM image of the $\mathrm{BaTiO}_{3}$ nanocrystal cluster sample. The clusters are obtained by sintering $100 \mathrm{~nm}$ cubic phase $\mathrm{BaTiO}_{3}$ nanocrystals at $1000{ }^{\circ} \mathrm{C}$ for one hour and rapidly cooling the nanocrystals in deionized water. The sintering converts the crystal structure from cubic phase to tetragonal phase while forming clusters with large size variations.

distance (30 $\mu \mathrm{m}$ in this experiment) and the reference is turned on.

The camera used in the experiment is a thermalelectrically cooled CCD camera with $6.8 \mu \mathrm{m}$ pixels and $7 \mathrm{e}^{-}$read noise. The quantum efficiency $(\mathrm{QE})$ of the image sensor is approximately $50 \%$ at $400 \mathrm{~nm}$ wavelength. The digitization sensitivity of the camera is factory calibrated to $3 \mathrm{e}^{-}$per digit. The camera is cooled down to $35{ }^{\circ} \mathrm{C}$ below the ambient temperature throughout the experiment, although the dark current does not play a significant role in the noise figure. We set the intensity of the hologram reference to at least 100 times stronger than the read noise of the CCD so that the read noise does not contribute significantly to the overall noise.

The digital image captured by the CCD camera is reconstructed numerically to obtain the electric field of the objective wave at the sample plane. Because the reference is introduced at near-zero angle with respect to the optical axis, we treat the hologram as in-line. The reconstruction algorithm is essentially a convolution between the holographic image intensity and the system impulse function [24]:

$$
\Gamma(\xi, \eta, \zeta)=h(x, y) * g(x, y ; \zeta),
$$

where $\Gamma(\xi, \eta, \zeta)$ is the reconstructed 3D image intensity at object coordinate $(\xi, \eta, \zeta), h(x, y)$ is the intensity of the holographic image captured from the CCD at hologram coordinate $(x, y)$, and $g(x, y ; \zeta)$ is the system impulse function at object coordinate $\zeta$ along the optical axis. This convolution is implemented as a sequence of fast Fourier transforms (FFTs) and inverse FFTs. In our configuration, the aspheric objective is not designed to accommodate the thick filter F2 (1.7 mm thickness), which causes significant spherical aberration in the image. To compensate for such aberrations, we computationally generate the impulse function $g(x, y ; \zeta)$ through ray tracing with precise design parameters published by the manufacturer. With this approach, we are able to completely 
cancel the aberration effect and obtain a reconstructed image with near diffraction-limited performance.

\section{Results}

As indicated in previous sections, imaging with second harmonic signals often involves a very low photon count. Thus an accurate estimate of signal levels for quantifying the SNR becomes difficult because of the intensity fluctuations due to shot noise. Since the signal level is directly proportional to the number of pulses integrated, we estimate the signal intensity at an integration of $n_{1}$ pulses through an image of $n_{2}$ pulses by $I_{1}=n_{1} I_{2} / n_{2}$, where $n_{2} \gg n_{1}$. An on-focus (direct) second harmonic image of the nanocrystal clusters is shown in Fig. 4. The image is acquired with an integration of 100 pulses and serves as an accurate measurement of signal intensity. For the ease of discussions, we assign each major object with a unique identification number.

To compare the performance of direct and holographic imaging, we capture direct and holographic images under identical conditions at integrations of 1, 5, and 10 pulses, which are shown in Fig. 5. Here we note that the experiments at different integration times are to investigate the imaging characteristics as a function of signal intensity rather than the integration time itself. The femtosecond laser pulses exclude the use of spatial filters and result in imperfections in the reference. Consequently, although the axial location ( $z$ coordinate) of the sample is accurately measured, we find it necessary to numerically reconstruct a stack of images along the $z$ axis within a $20 \mu \mathrm{m}$ range to find the best focus (location with the highest image intensity) for the holographic reconstruction. Because of the large dynamic range, the as-is image reconstructed from the hologram cannot be visualized with necessary clarity. Thus for visualization purpose, we histogram stretch every picture in Fig. 5 so that the darkest $0.01 \%$ pixels are black

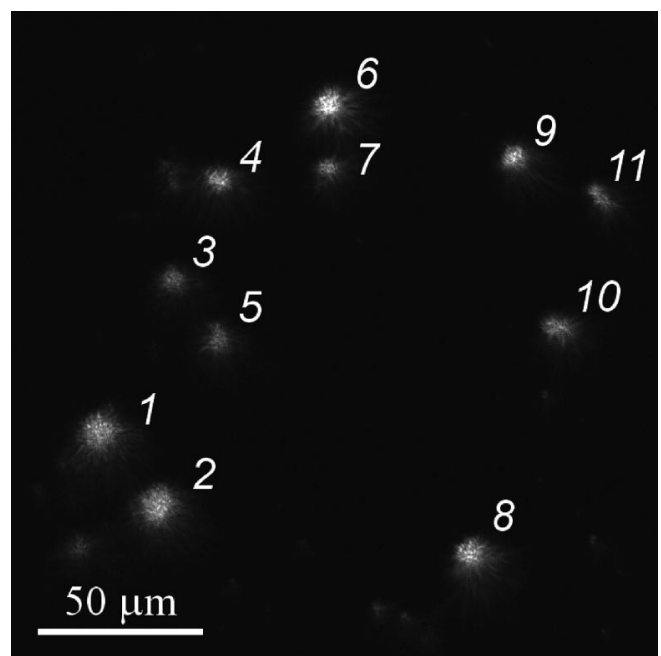

Fig. 4. Second harmonic on-focus image of the nanocrystal clusters obtained directly from the CCD at an integration of 100 laser pulses. The objects are numbered for ease of discussion. and the brightest $0.01 \%$ pixels are white. The effect of aberration compensation is clearly visible, as the nebulous images in the direct picture become much sharper in the reconstructed holographic image, revealing the fine structure of the nanocrystal clusters through a random phase-matching effect. Figure 6 shows a magnified image of object 7 , which is the smallest object among all identified objects. The remarkable compactness of the images in Figs. 6(d)6(f) suggests that the reconstruction is true focal images instead of speckles from off-focus objects. Figure 6 also shows a clear gain in SNR through reference biasing and aberration compensation.

Walking through the reconstructed image stack along the $z$ axis reveals the axial intensity profile of the holographic reconstruction, which is plotted in Fig. 7. The plot is obtained by averaging the normalized image intensity as a function of the $z$ coordinate over 50 randomly picked bright pixels at local maxima in Fig. 5(f). The position at which Fig. 5 is obtained is set to $z=0$. The inset in Fig. 7 shows a plot for a typical individual intensity profile. Figure 7 confirms that the images shown in Fig. 5 are true focal images. On average, the depth-of-focus of the reconstructed images is approximately $4.5 \mu \mathrm{m}$. The depthof-focus of an individual, small object is roughly $2.5 \mu \mathrm{m}$. Because of the size of the nanocrystal clusters (several micrometers), individual profiles peak at slightly different $z$ coordinates, resulting in a broadened mean intensity profile.

Because of the obvious difficulty in defining an image center for each object, we define the signal intensity for every image spot as the mean intensity for pixels with intensity above $50 \%$ of the local intensity maximum. As stated previously, to minimize the influence of the shot noise and to obtain accurate measurements, this procedure is performed only on an image of 100-pulse integration, and the intensity is then linearly scaled back to the proper integration of 1,5 , and 10 pulses. This approach is applied to both direct and holographic images to obtain signal intensities $\left\langle I_{i}^{(D)}\right\rangle$ and $\left\langle I_{i}^{(H)}\right\rangle$, respectively, where we calculate $\left\langle I_{i}^{(H)}\right\rangle=\left\langle Q_{i}^{(H)}\right\rangle / I_{R}$ from the mean intensity $\left\langle Q_{i}^{(H)}\right\rangle$ directly recovered from the hologram recorded with reference intensity $I_{R}$.

We quantify the gain of signal intensity from aberration compensation, $\gamma=\left\langle I_{i}^{(H)}\right\rangle /\left\langle I_{i}^{(D)}\right\rangle$, by a joint approach of ray tracing and wave propagation in the Fresnel regime. The value of $\gamma$ is a function of the objective NA, since a larger NA results in higher aberrations and thus a higher gain when the aberration is canceled. In this particular configuration where a NA of 0.45 is achieved (limited by the size of the objective), our numerical investigations show that the gain in signal intensity through aberration compensation is $\gamma=1.63$. If we increase the objective NA to 0.63 while all other parameters remain constant, the gain becomes $\gamma=3.0$.

Taking the shot noise into account, we now define the SNR for the direct images as 


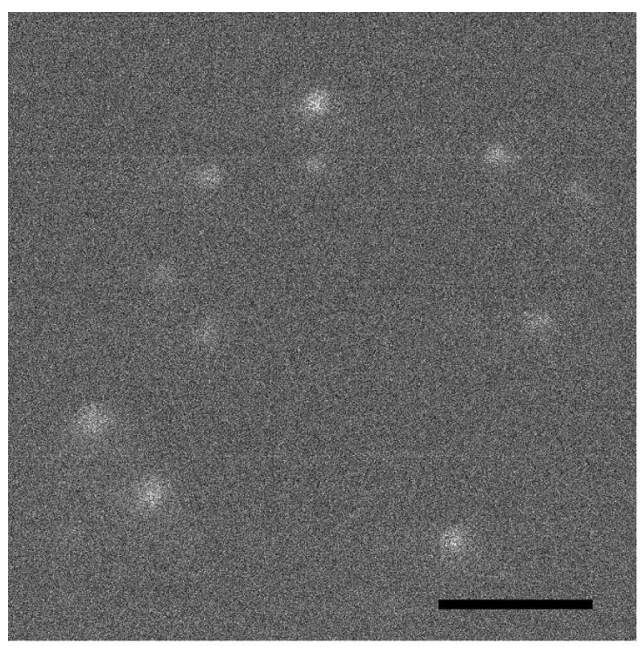

(a)

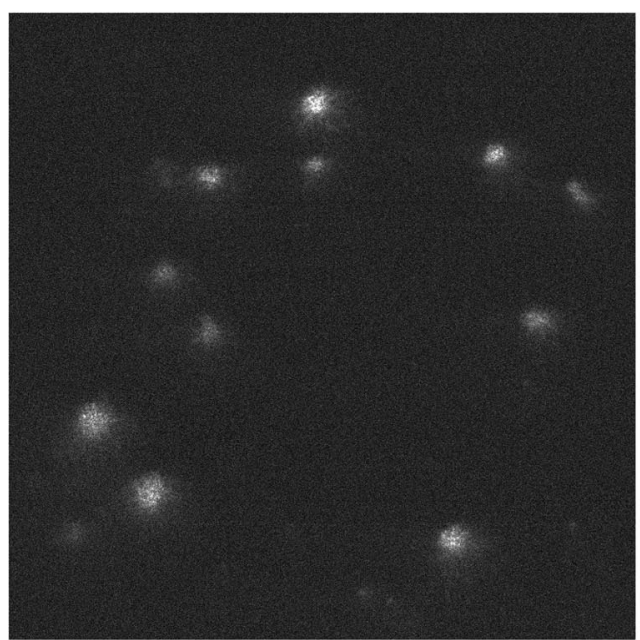

(b)

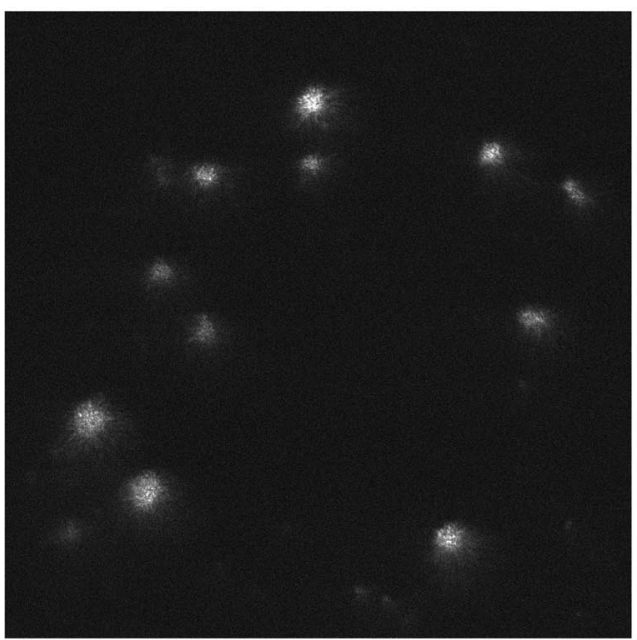

(c)

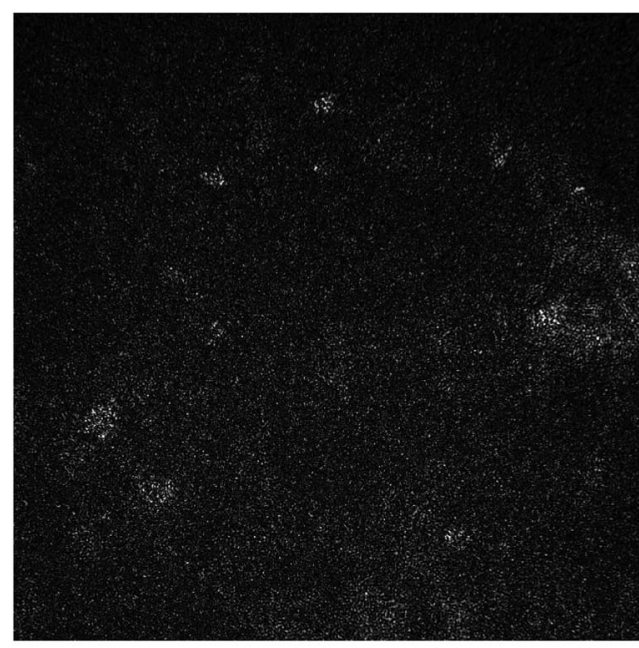

(d)

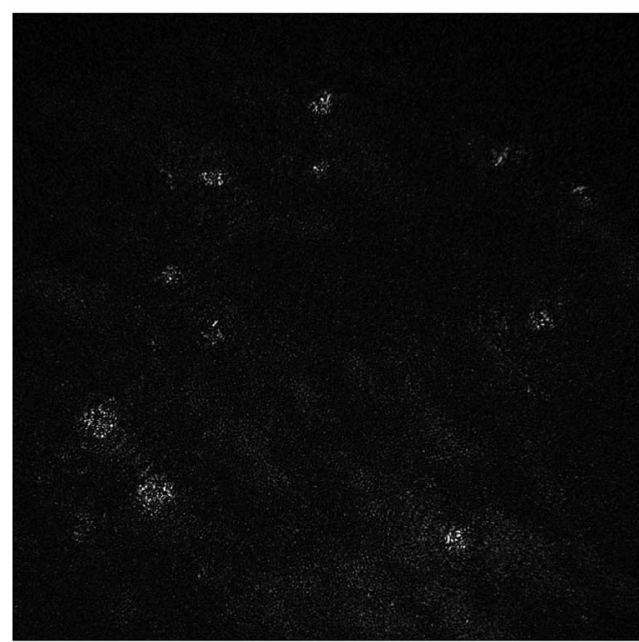

(e)

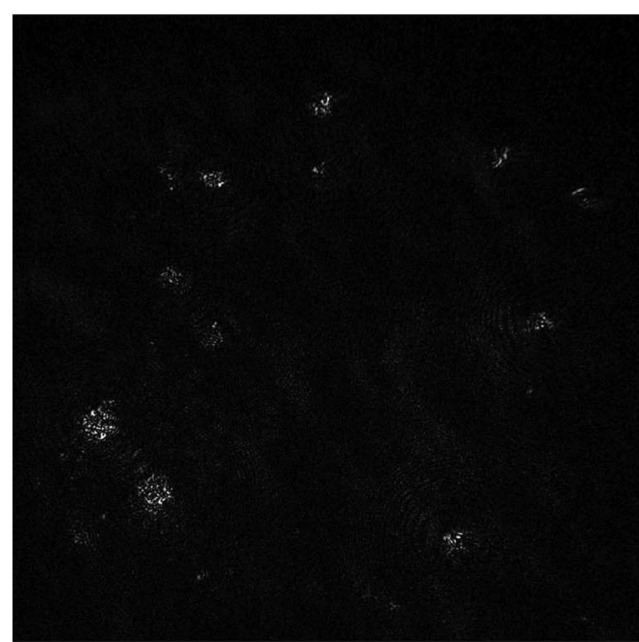

(f)

Fig. 5. Comparison between direct imaging and holographic reconstruction. (a), (c), (e) Direct imaging of 1, 5, and 10 pulses, respectively. (b), (d), (f) Holographic reconstruction of 1, 5, and 10 pulses, respectively. The effect of aberration compensation is clear. For consistent visualization, all images are histogram stretched so that the darkest $0.01 \%$ pixels are black and the brightest $0.01 \%$ pixels are white. The scale bar is $50 \mu \mathrm{m}$. 


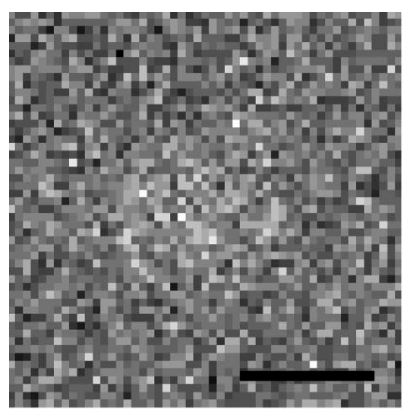

(a)

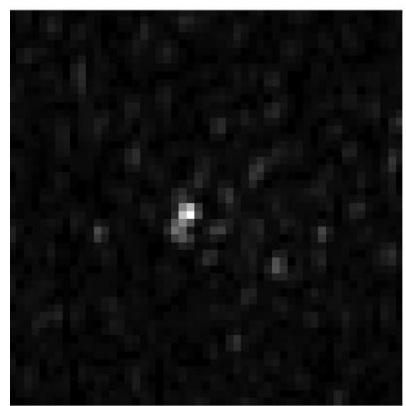

(d)

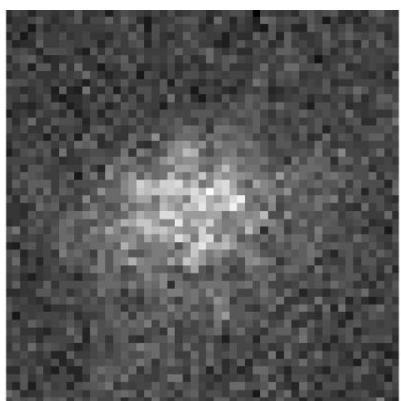

(b)

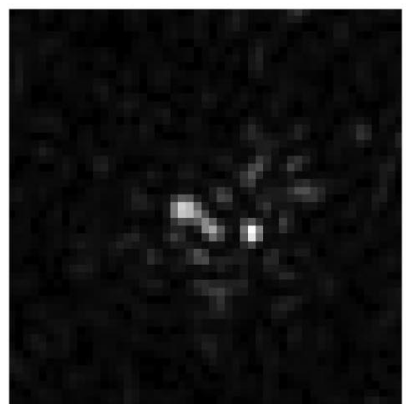

(e)

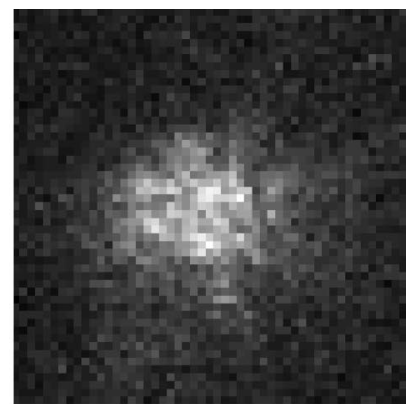

(c)

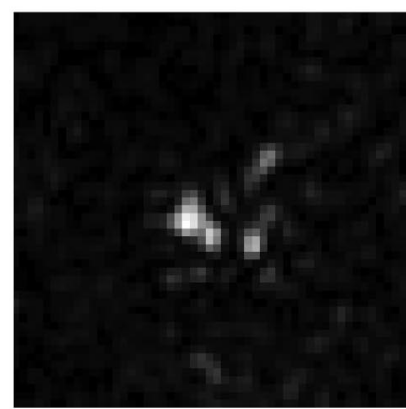

(f)

Fig. 6. Magnified images of object 7. (a), (b), (c) Direct imaging of 1, 5, and 10 pulses, respectively. (d), (e), (f) Holographic reconstruction of 1,5 , and 10 pulses, respectively. The scale bar is $5 \mu \mathrm{m}$.

$$
\mathrm{SNR}^{(D)}=\frac{\left\langle I_{i}^{(D)}\right\rangle \cdot \Delta}{\sqrt{\left\langle I_{i}^{(D)}\right\rangle \cdot \Delta+\left(N^{(D)} \cdot \Delta\right)^{2}}},
$$

where $\Delta$ is the digitization sensitivity of the CCD camera in photons per digit, and $N^{(D)}$ is the device noise after digitization in the direct image. Here we ignore the effect of $\mathrm{QE}$, which only affects the absolute sensitivity but not the SNR at the same level of

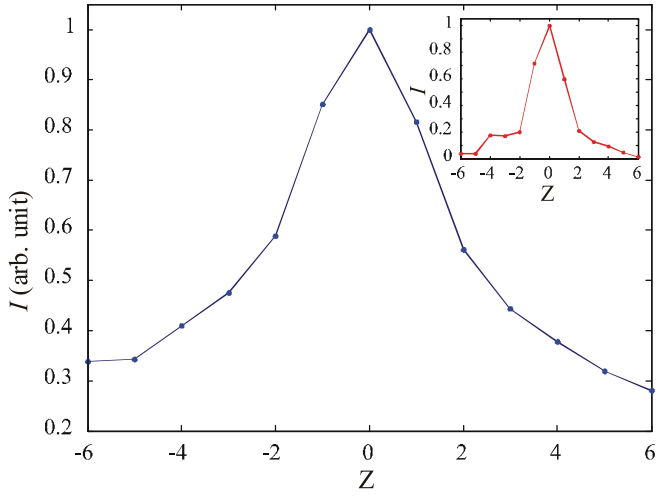

Fig. 7. (Color online) Normalized axial intensity profile of the holographic reconstruction. The profile is obtained by averaging the normalized image intensity as a function of the $z$ coordinate over 50 randomly picked bright pixels at local maxima in Fig. 5(f). The inset shows a typical profile of an individual image. The solid dots are experimental data, and the lines are a visual guide. On average, the depth-of-focus of the reconstructed images is approximately $4.5 \mu \mathrm{m}$. The depth-of-focus of an individual, small object is roughly $2.5 \mu \mathrm{m}$. The slightly broadened mean profile is due to the size of the nanocrystal clusters. $\left\langle I_{i}^{(D)}\right\rangle$. At the integration time used in this experiment, the read noise of the CCD camera dominates $N^{(D)}$.

The bias provided by the reference beam in $\mathrm{H}^{2}$ renders the device noise negligible, and as such, we obtain a shot-noise-limited device for all signal levels. Therefore the SNR for the holographic reconstruction is simply (again ignoring $\mathrm{QE}$ )

$$
\operatorname{SNR}^{(H)}=\sqrt{\left\langle I_{i}^{(H)}\right\rangle \cdot \Delta} .
$$

The overall gain in the SNR through holography over direct imaging is then

$$
G_{\mathrm{SNR}}=\frac{\mathrm{SNR}^{(H)}}{\operatorname{SNR}^{(D)}}=\sqrt{\gamma}\left(1+\frac{N^{(D)} \cdot \Delta}{\sqrt{I_{i}^{(D)}}}\right) .
$$

Given that $\left\langle I_{i}^{(D)}\right\rangle \approx 2.5$ photons $/$ pixel $/$ pulse, $\Delta \approx 3$ photons digit, and $N^{(D)} \approx 2.0$ in the camera used in this experiment, the measured SNR of the identified objects is listed in Table 1 . The fluctuations in $G_{\mathrm{SNR}}$ for each individual object are a result of the extended object size, whose scattering wavefront (at second harmonic frequency) deviates from that of a spherical wave [25]. In Fig. 8(a), we plot the SNR for both direct and holographic images, in red and blue, respectively, as a function of the number of integrated pulses. The empty triangles represent the measured data points, solid triangles with lines show the mean values of the measurement, and the dashed lines are theoretical predictions from Eqs. (3) and (4). The gain in SNR, $G_{\mathrm{SNR}}$, is plotted in Fig. 8(b) as a function of the num- 
Table 1. SNR of the Identified Objects ${ }^{a}$

\begin{tabular}{|c|c|c|c|c|c|c|c|c|c|c|c|}
\hline \multirow[b]{2}{*}{ Exposure } & \multicolumn{11}{|c|}{ Object } \\
\hline & 1 & 2 & 3 & 4 & 5 & 6 & 7 & 8 & 9 & 10 & 11 \\
\hline \multicolumn{12}{|l|}{1 Pulse } \\
\hline $\mathrm{D}$ & 1.1 & 1.2 & 0.9 & 1.1 & 0.9 & 1.4 & 1.0 & 1.2 & 1.2 & 1.0 & 0.9 \\
\hline $\mathrm{H}$ & 4.6 & 4.8 & 4.3 & 3.8 & 5.0 & 3.9 & 5.0 & 4.3 & 4.3 & 6.8 & 4.7 \\
\hline \multicolumn{12}{|l|}{5 Pulses } \\
\hline D & 4.4 & 4.5 & 3.6 & 4.3 & 3.7 & 5.1 & 4.1 & 4.6 & 4.6 & 4.0 & 3.7 \\
\hline $\mathrm{H}$ & 9.5 & 10.0 & 8.8 & 7.8 & 10.3 & 8.1 & 10.4 & 8.8 & 8.9 & 14.1 & 9.7 \\
\hline \multicolumn{12}{|l|}{10 Pulses } \\
\hline $\mathrm{D}$ & 7.0 & 7.3 & 6.0 & 7.0 & 6.0 & 8.2 & 6.6 & 7.4 & 7.5 & 6.5 & 6.1 \\
\hline $\mathrm{H}$ & 12.2 & 12.8 & 11.2 & 10.0 & 13.1 & 10.4 & 13.3 & 11.3 & 11.4 & 18.0 & 12.4 \\
\hline
\end{tabular}

${ }^{a} \mathrm{D}$, direct imaging; $\mathrm{H}$, holographic reconstruction.

ber of integrated pulses, where empty circles are measurement data points, solid circles with lines are the mean value, and the dashed line is the theoretical

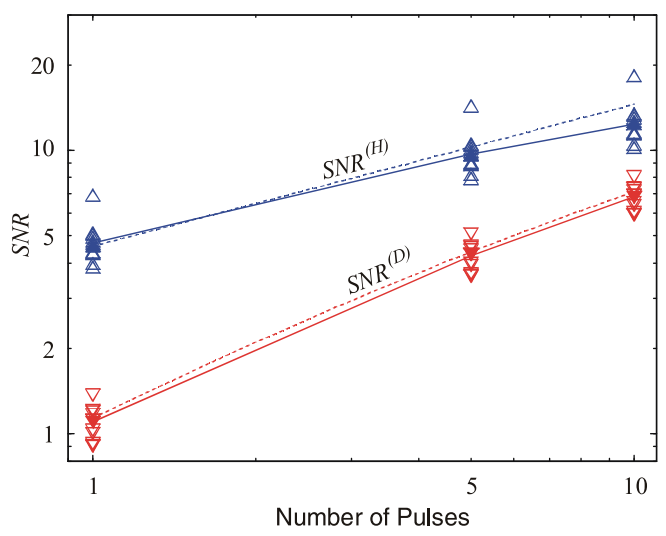

(a)

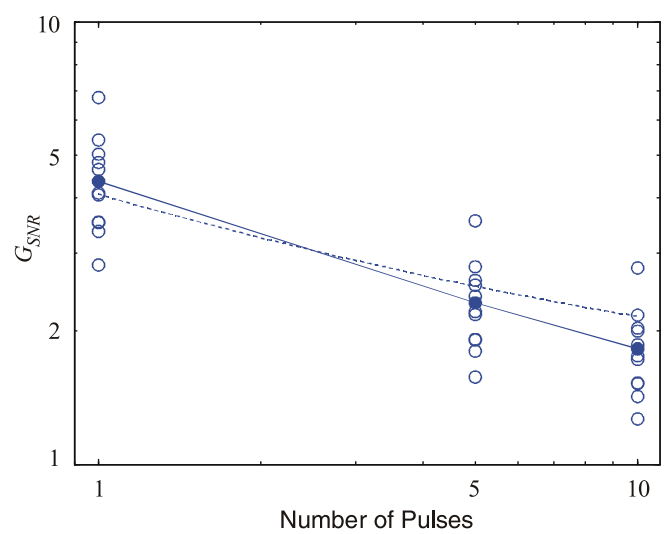

(b)

Fig. 8. (Color online) SNR characteristics of direct and reconstructed holographic images. (a) SNR of direct (down triangles) and holographic (up triangles) images. The empty triangles represent the measurement data points, solid triangles with lines show the mean values of the measurement, and the dashed lines are theoretical predictions from Eqs. (3) and (4). (b) Gain of SNR by holography over direct imaging. Empty circles are measurement data points, solid circles with line are the mean value, and the dashed line is the theoretical gain calculated from Eq. (5).
$G_{\mathrm{SNR}}$ calculated from Eq. (5). Due to the uncontrolled air flow in the laboratory environment, the value of $G_{\mathrm{SNR}}$ at longer integration falls slightly below what is predicted by Eq. (5).

\section{Conclusion}

Second harmonic generation provides a unique means to achieve contrast in the coherent domain, enabling the use of holography in contrast imaging. We show that a new holographic principle, harmonic holography $\left(\mathrm{H}^{2}\right)$, is promising for constructing an ultrafast four-dimensional contrast microscope with high spatial and temporal resolution. $\mathrm{H}^{2}$ records holograms using second harmonic signals scattered from SHG nanocrystals and an independently generated second harmonic reference. Our experiments show that the technique of $\mathrm{H}^{2}$ has unique advantages over direct imaging, including numerical aberration compensation and device noise canceling. The experimental data for the SNR performance is in very good agreement with the theoretical predictions. $\mathrm{H}^{2}$ has proved to be a powerful technique to achieve aberration-free, shot-noise-limited performance with low photon count signals, laying a sound basis for molecular biomedical imaging applications. The optimal performance can be achieved through the use of high NA objectives (which bear significant aberrations when used to image a volume) and small pixel, fast frame rate cameras (which typically have high device noise). This principle can also be extended to use other coherent optical process, such as sum frequency generation, difference frequency generation, and coherent anti-Stokes Raman scattering.

This work is supported by the DARPA Center for Optofluidic Integration at Caltech. The authors thank Scott Fraser, John Hong, Periklis Pantazis, and James Adleman for extremely helpful discussions.

\section{References}

1. G. M. Whitesides, "The 'right' size in nanobiotechnology," Nat. Biotechnol. 21, 1161-1165 (2003).

2. E. B. Brauns, M. L. Madaras, R. S. Coleman, C. J. Murphy, and M. A. Berg, "Complex local dynamics in DNA on the picosecond 
and nanosecond time scales," Phys. Rev. Lett. 88, 158101 (2002).

3. R. Gilmanshin, S. Williams, R. H. Callender, W. H. Woodruff, and R. B. Dyer, "Fast events in protein folding: relaxation dynamics of secondary and tertiary structure in native apomyoglobin," Proc. Natl. Acad. Sci. USA 94, 3709-3713 (1997).

4. D. Gabor, "A new microscopic principle," Nature 161, 777-778 (1948).

5. E. N. Leith and J. Upatniek, "Wavefront reconstruction with diffused illumination + 3-dimensional objects," J. Opt. Soc. Am. 54, 1295-1301 (1964).

6. E. N. Leith, J. Upatniek, and K. A. Haines, "Microscopy by wavefront reconstruction,” J. Opt. Soc. Am. 55, 981-986 (1965).

7. U. Schnars and W. Juptner, "Direct recording of holograms by a ccd target and numerical reconstruction," Appl. Opt. 33, 179-181 (1994).

8. I. Yamaguchi and T. Zhang, "Phase-shifting digital holography," Opt. Lett. 22, 1268-1270 (1997).

9. E. Cuche, P. Marquet, and C. Depeursinge, "Simultaneous amplitude-contrast and quantitative phase-contrast microscopy by numerical reconstruction of fresnel off-axis holograms," Appl. Opt. 38, 6994-7001 (1999).

10. F. Dubois, L. Joannes, and J. C. Legros, "Improved threedimensional imaging with a digital holography microscope with a source of partial spatial coherence," Appl. Opt. 38, 7085-7094 (1999).

11. P. Marquet, B. Rappaz, P. J. Magistretti, E. Cuche, Y. Emery, T. Colomb, and C. Depeursinge, "Digital holographic microscopy: a noninvasive contrast imaging technique allowing quantitative visualization of living cells with subwavelength axial accuracy," Opt. Lett. 30, 468-470 (2005).

12. L. Miccio, D. Alfieri, S. Grilli, P. Ferraro, A. Finizio, L. De Petrocellis, and S. D. Nicola, "Direct full compensation of the aberrations in quantitative phase microscopy of thin objects by a single digital hologram," Appl. Phys. Lett. 90, 041104 (2007).

13. L. Toth and S. A. Collins, "Reconstruction of a 3-dimensional microscopic sample using holographic techniques," Appl. Phys. Lett. 13, 7-9 (1968).

14. W. Xu, M. H. Jericho, H. J. Kreuzer, and I. A. Meinertzhagen, "Tracking particles in four dimensions with in-line holographic microscopy," Opt. Lett. 28, 164-166 (2003).

15. T. Zhang and I. Yamaguchi, "Three-dimensional microscopy with phase-shifting digital holography," Opt. Lett. 23, 12211223 (1998).

16. I. Yamaguchi, J. Kato, S. Ohta, and J. Mizuno, "Image formation in phase-shifting digital holography and applications to microscopy," Appl. Opt. 40, 6177-6186 (2001).

17. Y. Pu and H. Meng, "Four-dimensional dynamic flow measurement by holographic particle image velocimetry," Appl. Opt. 44, 7697-7708 (2005).

18. D. Gerlich and J. Ellenberg, "4D imaging to assay complex dynamics in live specimens," Nat. Cell Biol. 5, S14-S19 (2003).

19. P. J. Campagnola and L. M. Loew, "Second-harmonic imaging microscopy for visualizing biomolecular arrays in cells, tissues and organisms," Nat. Biotechnol. 21, 1356-1360 (2003).

20. A. Andreoni, M. Bondani, M. A. C. Potenza, and Y. N. Denisyuk, "Holographic properties of the second-harmonic cross correlation of object and reference optical wave fields," J. Opt. Soc. Am. B 17, 966-972 (2000).

21. G. Pedrini, S. Schedin, and H. J. Tiziani, "Aberration compensation in digital holographic reconstruction of microscopic objects," J. Mod. Opt. 48, 1035-1041 (2001).

22. F. Charriere, T. Colomb, F. Montfort, E. Cuche, P. Marquet, and C. Depeursinge, "Shot-noise influence on the reconstructed phase image signal-to-noise ratio in digital holographic microscopy,” Appl. Opt. 45, 7667-7673 (2006).

23. R. W. Boyd, Nonlinear Optics (Academic, 2002).

24. U. Schnars and W. P. O. Juptner, "Digital recording and numerical reconstruction of holograms," Meas. Sci. Technol. 13, R85-R101 (2002).

25. Y. Pu and H. Meng, "Intrinsic aberrations due to mie scattering in particle holography," J. Opt. Soc. Am. A 20, 1920-1932 (2003). 